\title{
A Comparison of Bound-Constrained and Positivity-Constrained Optimization Method to Estimate Head Tissue Conductivities by Scalp Voltage Information
}

Preliminary Communication

\author{
Taweechai Ouypornkochagorn \\ Faculty of Engineering, Srinakharinwirot University, Thailand \\ taweechai@g.swu.ac.th
}

\section{Chollanant Khattiyawech}

Faculty of Engineering, Srinakharinwirot University, Thailand chollanant.13@gmail.com

\section{Natnicha Keatsiritawon \\ Faculty of Engineering, Srinakharinwirot University, Thailand natcha_k.natnicha@hotmail.com}

\begin{abstract}
Electrical Impedance Tomography (EIT) is a noninvasive method used to estimate the conductivity of head tissues. Estimation based on the unconstrained Gauss-Newton (GN) method is conventional, but it may result in negative-value or extraordinary high-value estimates, which are unexpected. In this study, the bound-constrained method and the positivity-constrained optimization method were investigated and compared to the unconstrained optimization method. A two-dimensional model was created for conductivity estimation containing five head tissues, i.e., the scalp, the skull, the cerebrospinal fluid (CSF), grey matter (GM), and white matter (WM). The results showed that the accuracy, the robustness, and the estimation convergence of the estimation of this approach were significantly improved by constraining. All unexpected values also disappeared. The investigation proved that very high sensitivity of the skull region caused the unexpected outcome of the unconstrained cases. This high sensitivity can be significantly reduced by constraining. However, a degree of estimation nonlinearity can be increased by constraining as well, causing some estimation accuracies in the case of the positivityconstrained optimization method to be poor. Therefore, it is recommended to use only the bound-constrained optimization method.
\end{abstract}

Keywords - bound-constrained optimization, electrical impedance tomography (EIT), head tissue conductivity, positivity-constrained optimization

\section{INTRODUCTION}

Head tissue conductivities are used in many medical applications, e.g. localization of cortical regions in human brain in response to brain stimulations [1], navigation in deep brain surgery [2], and electromagnetic imaging of the source of brain function [3]. Conventionally, only five tissues are of interest, i.e. the scalp, the skull, the cerebrospinal fluid (CSF), grey matter (GM), and white matter (WM). Many studies have discussed the conductivity of these tissues, as shown in Table 1. Samples of tissues are examined in the case of in vitro method. This method is invasive. The conductivities obtained by this method are commonly used as references in the present. Due to sample manipulation, the accuracy of the conductivity measurement of this method is still an issue of concern.
The obtained conductivity may be inaccurate by the influence of storing duration, air exposing, cooling, drying, or cleaning. In the case of in vivo method, it could be both invasive and noninvasive. To invasively measure the conductivity of the tissues situated in the deep region of the head, needle electrodes were invasively pierced into the deep region of the head [4], or intracerebral multicontact electrodes were implanted [5]. Tissue conductivities can be estimated non-invasively by magnetoencephalography (MEG) - a contactless method $[6,7]$, or by using the boundary voltage information of electroencephalography (EEG) $[6,8]$, or the electrical impedance tomography (EIT) technique $[9,10]$, which are much cheaper in terms of both operation and instrument costs. Interestingly, as shown in Table 1, the conductivities obtained by these methods are not significantly different. 
To estimate the conductivities by using scalp voltage information, the estimation based on the Gauss-Newton (GN) method is usually used to estimate the conductivities in conjunction with a subject head model. The estimation may be performed without using current or voltage source, i.e. EEG $[7,11]$, or by using the source, i.e. EIT $[9,10]$. The estimation based on EIT is most popular for obtaining generic conductivities. However, the reported conductivity values based on the GN method are unstable. Conductivity values may be negative, out of range reported by in vitro methods. To the best knowledge of the authors of this paper, there has been no successful conductivity estimation for the CSF, GM, and WM. This is due to susceptibility to noise and error of the conventional GN method. Noise could be instrumentation noise and physiological noise. Instrumentation noise occurs due to poor hardware design or the limitation of the hardware, e.g. the resolution of an analog-to-digital converter. Physiological noise is an irrelevant physiological response that cannot be isolated from the interested response. In the case of error, it could be a modeling error and a linearization error. This includes an error due to the selection of initial guesses for the estimation. Recently, the bound-constrained optimization method and the positivity-constrained optimization method have been proposed for EIT to improve the performance of image reconstruction [12]. The estimated pixel values were constrained to be only positive, resulting in fewer artifacts in images. Accordingly, the constrained method could be possibly used to estimate the conductivities with robustness to noise and error.

In this study, the head conductivities were estimated based on the EIT technique. Two constrained methods, i.e. the bound-constrained optimization and the positivity-constrained optimization method, were used to estimate the scalp, skull, CSF, GM, and WM conductivity. The conventional GN method which is an unconstrained optimization method was used in the comparison.

Table 1. Head tissue conductivities reported in the literature

\begin{tabular}{|c|c|c|c|}
\hline Tissues & $\begin{array}{l}\text { In vitro method } \\
\text { (S/M) }\end{array}$ & $\begin{array}{l}\text { In vivo and invasive method } \\
\text { (S/M) }\end{array}$ & $\begin{array}{l}\text { In vivo and noninvasive method } \\
\text { (S/M) }\end{array}$ \\
\hline Scalp & $0.22^{[13]}, 0.4348^{[14]}$ & & $\begin{array}{c}0.315^{[7]}, 0.3-0.4^{[9]}, 0.3322-0.4277^{[6]} \\
0.58^{[10]}\end{array}$ \\
\hline Skull & $\begin{array}{c}0.0049-0.0095^{[15]}, 0.0131^{[8]} \\
0.0132-0.0324^{[16]} \\
0.0214-0.0801^{[17]}\end{array}$ & & $\begin{array}{c}0.0045-0.005^{[9]}, 0.0062-0.0113^{[6]} \\
0.008^{[10]}, 0.0093-0.015^{[11]} \\
0.015^{[8]}, 0.016^{[7]}\end{array}$ \\
\hline CSF & $1^{[18]}, 1.456-1.802^{[19]}$ & $1.25^{[4]}$ & \\
\hline GM & $0.33-0.43^{[18]}$ & $0.2849^{[4]}, 0.26^{[5]}$ & \\
\hline WM & $0.76^{[18]}$ & $0.2556^{[4]}, 0.17^{[5]}$ & \\
\hline
\end{tabular}

\section{METHODS}

\subsection{ELECTRICAL IMPEDANCE TOMOGRAPHY (EIT)}

EIT is a technique to image the conductivity distribution $\sigma$ of a measured subject as a conductive volume $\Omega$. Boundary voltage $V$, i.e. scalp voltage, is collected, while a small current $I_{e l}$ is injected at the volume boundary $\Omega_{\mathrm{el}}$. The system is governed by the following equations [20]:

$$
\begin{array}{lr}
\nabla \bullet(\sigma(x) \nabla u(x))=0, & x \in \Omega \\
\int_{e l} \sigma(x) \frac{\partial u(x)}{\partial v} d s=I_{e l}, & x \in \Omega_{e l} \\
\sigma(x) \frac{\partial u(x)}{\partial v}=0, & x \notin \Omega_{e l}
\end{array}
$$

where $x$ is a point in $\Omega, u$ is the potential distribution inside $\Omega$, and $z_{e l}$ is the contact impedance.

$$
u(x)+z_{e l} \sigma(x) \frac{\partial u(x)}{\partial v}=V, \quad x \in \Omega_{e l}
$$

\subsection{UNCONSTRAINED-OPTIMIZATION METHOD}

The scalp voltage $V$ is predicted by the EIT model:

$$
V=U(\sigma)+e
$$

where $U$ is the forward modeling function of conductivity distribution $\sigma$, and e is noise which is included the system error. $\hat{\sigma}$ is the estimate, where the given prediction voltages $U$ fit $V$, which is estimated by:

$$
\hat{\sigma}=\underset{\sigma}{\arg \min }\left\{\|V-U(\sigma)\|^{2}\right\}
$$


The iterative regularized Gauss-Newton (GN) method is usually used by:

$$
\hat{\sigma}_{i+1}=\hat{\sigma}_{i}+\left(J^{T} J+\lambda R^{T} R\right)^{-1} J^{T}\left(V-U\left(\hat{\sigma}_{i}\right)\right),
$$

where $J$ is the sensitivity matrix or the Jacobian matrix, i.e. $\partial U, R$ is the regularization matrix, $\lambda$ is the regularization parameter, and $i$ is the iteration index. Note that $\hat{\sigma}$ of the first iteration is a vector of initial guesses. In order to estimate the generic conductivity of each tissue, the sensitivity of each tissue region is averaged $[9,10]$.

\subsection{BOUND-CONSTRAINT OPTIMIZATION METHOD}

The scaled-logistic function is used to constrain $\sigma$, which is transformed to $\gamma$ :

$$
\gamma=-\kappa \log \left(\frac{t-p}{\sigma-p}-1\right) \text { or, } \sigma=p+\frac{t-p}{1+e^{-\gamma / \kappa}},
$$

where $t$ is the upper bound, $p$ is the lower bound, and $K$ is the relaxation factor. $t$ and $p$ are determined from the values obtained from the literature (Table 1). The sensitivity matrix of $\gamma$ is computed by [12]:

$$
J_{\gamma}=\partial_{\sigma} U(\sigma)(\sigma-p)(t-\sigma)(t-p)^{-1} \kappa^{-1}
$$

$\hat{\gamma}$ is then determined from:

$$
\hat{\gamma}_{i+1}=\hat{\gamma}_{i}+\left(J_{\gamma}^{T} J_{\gamma}+\lambda R^{T} R\right)^{-1} J_{\gamma}^{T}\left(V-U\left(\hat{\sigma}_{i}\right)\right) \text {. }
$$

Eventually, $\hat{\gamma}$ is reversely transformed to $\hat{\sigma}$ by (8).

\subsection{POSITIVITY-CONSTRAINT OPTIMIZATION METHOD}

The logarithmic function is used in this method instead of the scaled-logistic function to constrain the estimation to only the positive value by:

$$
\gamma=\kappa \log (\sigma) \text { or, } \sigma=e^{\gamma / \kappa}
$$

The sensitivity matrix of $\gamma$ is computed by [12]:

$$
J_{\gamma}=\partial_{\sigma} U(\sigma) \sigma \kappa^{-1}
$$

$\hat{\gamma}$ is determined by (10), and $\hat{\sigma}$ is then obtained by using (11).

\subsection{SIMULATION CONFIGURATION}

A 2D-circular model containing five layers (from the outermost layer to the innermost layer), i.e. the scalp, the skull, the CSF, GM and WM, and having 10,816 elements was used in the simulation (Fig. 1). The diameter was $170 \mathrm{~mm}$, and the tissue thicknesses, ordered from the outermost to the innermost, were $6.6 \mathrm{~mm}, 6.6 \mathrm{~mm}, 6.6 \mathrm{~mm}, 8.2 \mathrm{~mm}$, and $57 \mathrm{~mm}$, respectively. Sixteen electrodes were attached, where 192 nearest-neighbor measurements were collected. $1 \mathrm{~mA}_{\text {peak-peak }}$ current was diametrically injected to a pair of electrodes. There were 16 diametrically current patterns used. Small noise of a $50 \mathrm{~dB}$ signal-to-noise ratio was added to the measurements. The simulated conductivities were $0.58 \mathrm{~S} / \mathrm{m}, 0.008 \mathrm{~S} / \mathrm{m}, 1.802 \mathrm{~S} / \mathrm{m}$, $0.2849 \mathrm{~S} / \mathrm{m}$, and $0.2556 \mathrm{~S} / \mathrm{m}$ for the scalp, the skull, the CSF, the GM layer, and WM, respectively. Thirty initial guesses used in the simulation were randomly selected from the values shown in Table 1. Estimation using the unconstrained GN method was compared to that using the bound-constrained and the positivityconstrained GN methods. The regularization parameter was set to $1 \times 10^{-4}$ and the relaxation factor was set to one for all cases. In the case of the boundconstrained method, the upper bound was set to 2 $\mathrm{S} / \mathrm{m}$ and the lower bound was set to $0 \mathrm{~S} / \mathrm{m}$, covering the reported values shown in Table 1. The number of iterations was 15, which checked that all estimations stagnated. The Jacobian matrix was updated in every iteration with the estimated conductivities of the previous iteration. EIDORS software (http://eidors3d. sourceforge.net) was used to solve the EIT forward problem. Relative error was used to determine the accuracy of the methods, as shown in (13). A small value of relative error was expected.

$$
\text { RelativeError }=\frac{\|V-U(\sigma)\|}{\|V\|} .
$$

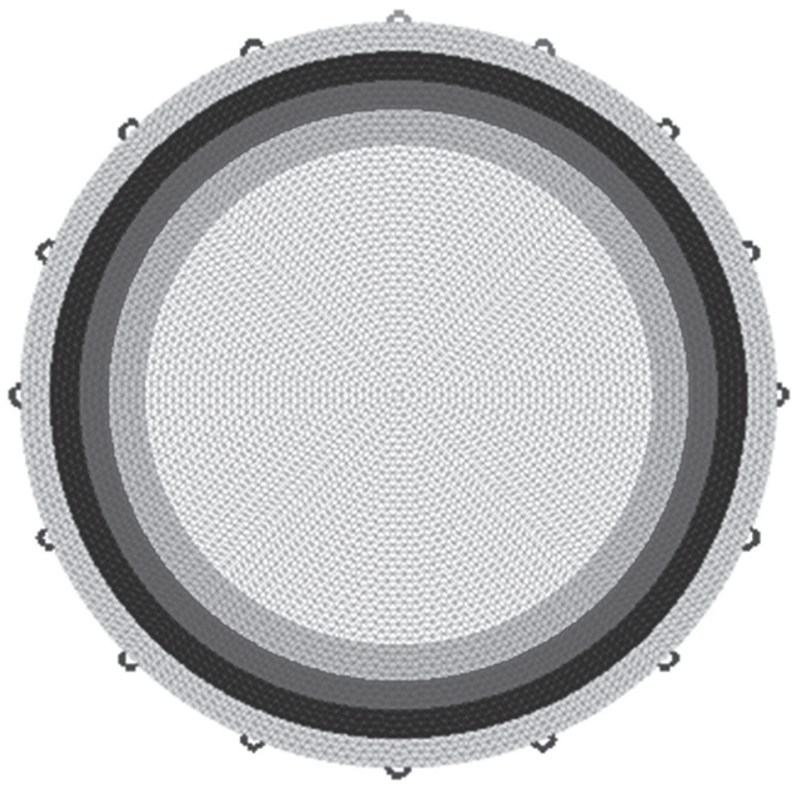

Fig. 1. The model used in the simulation containing (from the outermost layer to the innermost layer) the scalp, the skull, the CSF, the GM, and the WM 


\section{RESULTS}

Estimation results are shown in Table 2 and Fig. 2. In Table 2, the displayed estimates have excluded the estimates whose conductivity value is negative or greater than $2 \mathrm{~S} / \mathrm{m}$, which is within range of the reported values (as shown in Table 1). Similarly to Fig. 2, the estimates where the conductivity value is very large or negative are not displayed. It is clearly seen that the use of the unconstrained optimization method, which is the conventional method, resulted in lots of negative or over-range estimates, in particular, the estimates of the GM and the WM conductivity. 70\% of these estimates were negative. The values can be down to $-233 \mathrm{~S} / \mathrm{m}$ and up to $265 \mathrm{~S} / \mathrm{m}$. The over-range estimates occurred when using the positivity-constrained optimization method as well, in particular, those of the CSF conductivities. $66.7 \%$ of these estimates were over-ranged. The values can be up to $320 \times 10^{6} \mathrm{~S} / \mathrm{m}$. It is interesting that these unexpected values did not occur when the boundconstrained optimization method was used.

When the unexpected values were not taken into account, estimation for the scalp conductivity was satisfactory for all methods (the worst error was only $0.7 \%$ ). Estimation of the skull and the WM conductivity was plausible for both the unconstrained and the bound-constrained optimization method. More specifically, the estimation accuracy for the skull conductivity of the bound-constrained optimization method was much better than that of the unconstrained optimization method, i.e. by $11.25 \%$. Estimation of the CSF conductivity in the case of using the bound-constrained optimization method showed only marginal accuracy, but this accuracy was better than the other methods. Only the estimation for the GM conductivity in the case of using the unconstrained (with the exclusion of negative estimates) and the positivity-constrained optimization method was more accurate than that obtained by using the boundconstrained optimization method.

The convergences of the estimations of all methods are shown in figures 3-5. It is obvious that the unconstrained optimization method was less robust to the selection of initial guesses than the other constrained optimization methods. Many error norms were large and required more iterations to restagnate. Overall, it required approximately 8 iterations to stagnate. The convergence rate of the positivityconstrained optimization method proved to be slightly more plausible than that of the bound-constrained optimization method, although some estimation cases were larger in error. The estimations stagnated at approximately the $4^{\text {th }}$ iteration for both constrained optimization methods.

The susceptibility to the selection of the initial guesses can be determined from Fig. 6. In general, only a slight difference in estimation errors can be noticed from all methods. Mostly, the error ranged between 0.0032 and 0.0049 . Only three cases of the unconstrained optimization method and one case of the positivity-constrained optimization method were beyond this range, i.e. greater than 0.49 . This indicates higher robustness to the initial guess selection of the bound-constrained optimization method.

Table 2. Conductivity estimation by the unconstrained and the constrained optimization methods

\begin{tabular}{|c|c|c|c|c|}
\hline Tissues & $\begin{array}{c}\text { Simulated exact } \\
\sigma(\mathrm{S} / \mathrm{M}) \\
\text { (Target values) }\end{array}$ & $\begin{array}{l}\text { Unconstrained optimization } \\
\text { method }^{\text {a }}\end{array}$ & $\begin{array}{l}\text { Bound-constrained } \\
\text { optimization method }^{\text {a }}\end{array}$ & $\begin{array}{l}\text { Positivity-constrained } \\
\text { optimization method }^{\text {a }}\end{array}$ \\
\hline Scalp & 0.58 & $0.5972 \pm 0.015^{b}$ & $0.5758 \pm 0.014$ & $0.5799 \pm 0.001^{f}$ \\
\hline Skull & 0.008 & $0.0069 \pm 0.001^{c}$ & $0.0082 \pm 0.001$ & $0.0420 \pm 0.186$ \\
\hline CSF & 1.802 & $1.2979 \pm 0.333^{d}$ & $1.6886 \pm 0.324$ & $1.4600 \pm 0.371^{\mathrm{g}}$ \\
\hline GM & 0.2849 & $0.2933 \pm 0.137^{e}$ & $0.8130 \pm 0.659$ & $0.2900 \pm 0.154$ \\
\hline WM & 0.2556 & $0.2543 \pm 0.079^{e}$ & $0.2332 \pm 0.353$ & $0.1405 \pm 0.108$ \\
\hline
\end{tabular}

${ }^{a}$ The value represented as the mean \pm standard.

${ }^{\mathrm{b}}$ The averaging has excluded 2 estimates over $2 \mathrm{~S} / \mathrm{m}$ (6.7\%).

' The averaging has excluded 2 negative estimates (6.7\%).

${ }^{d}$ The averaging has excluded 3 estimates over $2 \mathrm{~S} / \mathrm{m}(10.0 \%)$ and 1 negative estimate (3.3\%). e The averaging has excluded 21 negative estimates (70\%).

f The averaging has excluded 1 estimate over 2 S/m (3.3\%)

9The averaging has excluded 20 estimates over 2 S/m (66.7\%). 


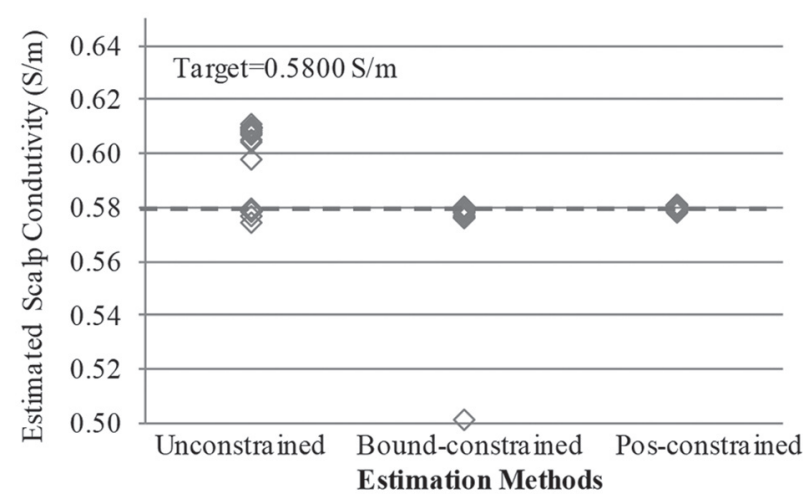

(a) Estimated Scalp Conductivities

(only the estimates ranging between 0.50 and 0.65 are displayed, i.e., 2 estimates of the unconstrained optimization method and 1 estimate of the positivity-constrained optimization method are excluded)

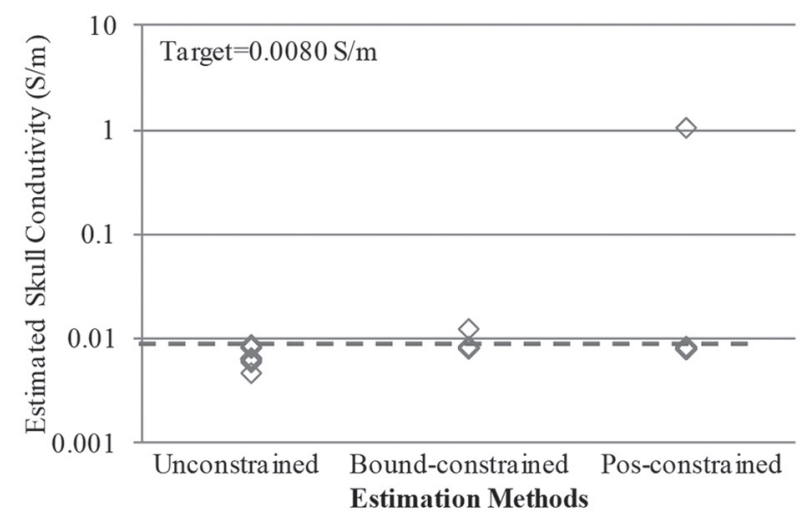

(b) Estimated Skull Conductivities (displayed on a log-scale, and only the estimates ranging between 0.001 and 10 are displayed, i.e., 2 estimates of the unconstrained optimization method are excluded)

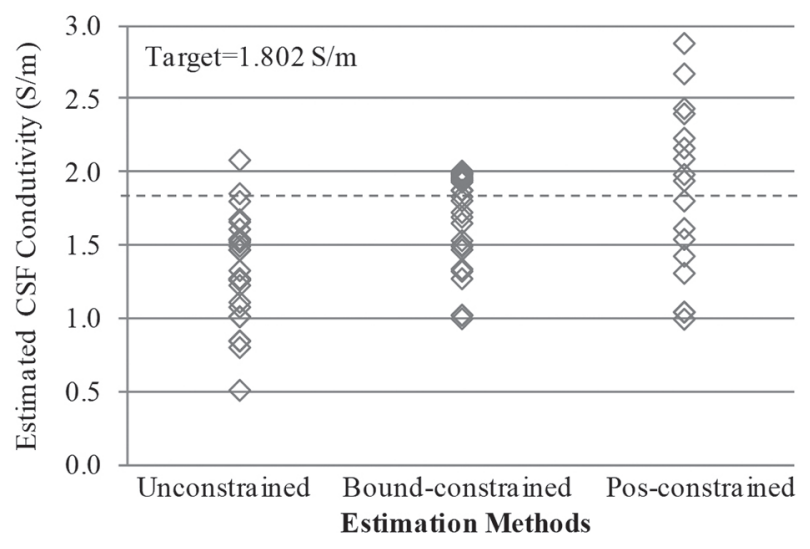

(c) Estimated CSF Conductivities (only the estimates ranging between 0 and 3 are displayed, i.e., 3 estimates of the unconstrained optimization method and 13 estimates of the positivityconstrained optimization method are excluded)

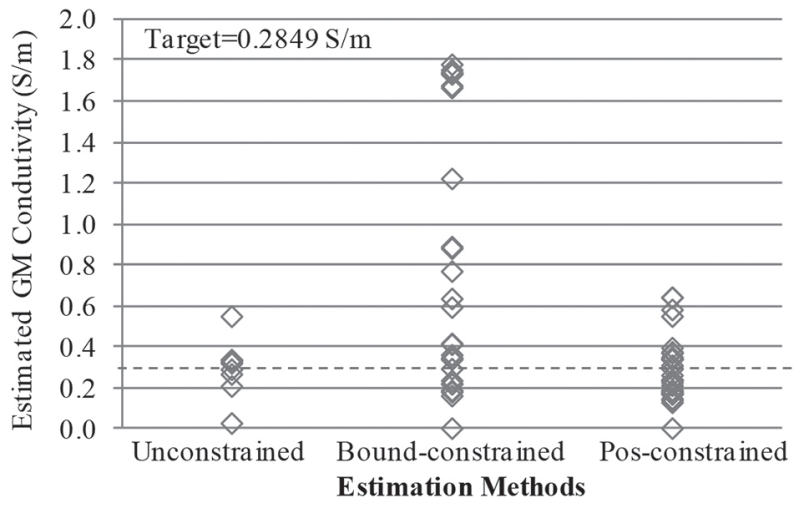

(d) Estimated GM Conductivities

(only the estimates ranging between 0 and 2 are displayed, i.e., 21 estimates of the unconstrained optimization method are excluded)

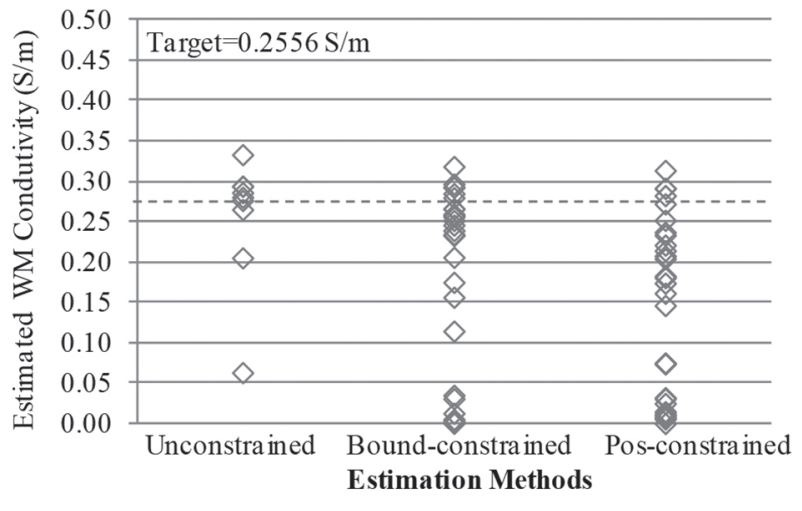

(e) Estimated WM Conductivities

(only the estimates ranging between 0 and 0.5 are displayed, i.e., 21 estimates of the unconstrained optimization method and 1 estimate of the boundconstrained optimization method are excluded)

Fig. 2. Estimation conductivities using the unconstrained, the bound-constrained, and the positivity-constrained optimization method with 30 initial guesses:

(a) scalp, (b) skull, (c) CSF, (d) GM, and (e) WM conductivities 


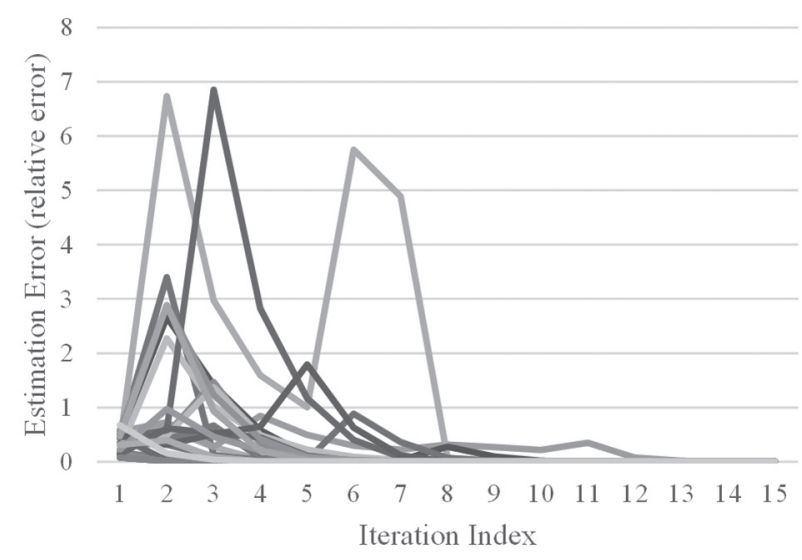

Fig. 3. Convergence of the estimation using the unconstrained optimization method over 30 initial guesses

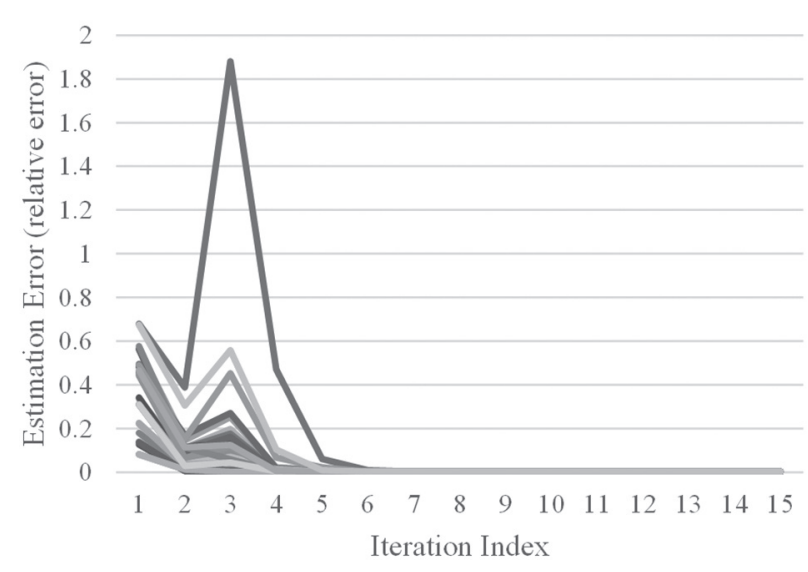

Fig. 5. Convergence of the estimation using the positivity-constrained optimization method over 30 initial guesses

\section{DISCUSSION}

According to the large number of unexpected estimates in the case of using the unconstrained optimization method in conjunction with poorer accuracies compared to other constrained optimization methods, the unconstrained optimization method is suitable only for scalp and skull conductivity estimation. However, there is still a $6.7 \%$ chance to have unexpected values. The bound-constrained optimization method is suitable for almost all cases, with the exception of GM conductivity estimation. The positivity-constrained optimization method is suitable to estimate the scalp, the GM, and the WM conductivities. However, there is still a $3.3 \%$ chance to have unexpected values.

Two additional analyses were carried out to investigate the condition number of the Jacobian matrices and the norm of element sensitivities in each of the tissue regions, as shown in tables 3 and 4 . It is obvious that constraining can decrease the condition number by 31 76 times (Table 3). The condition number of the positivityconstrained optimization method is 2.4 times less than that of the bound-constrained optimization method.

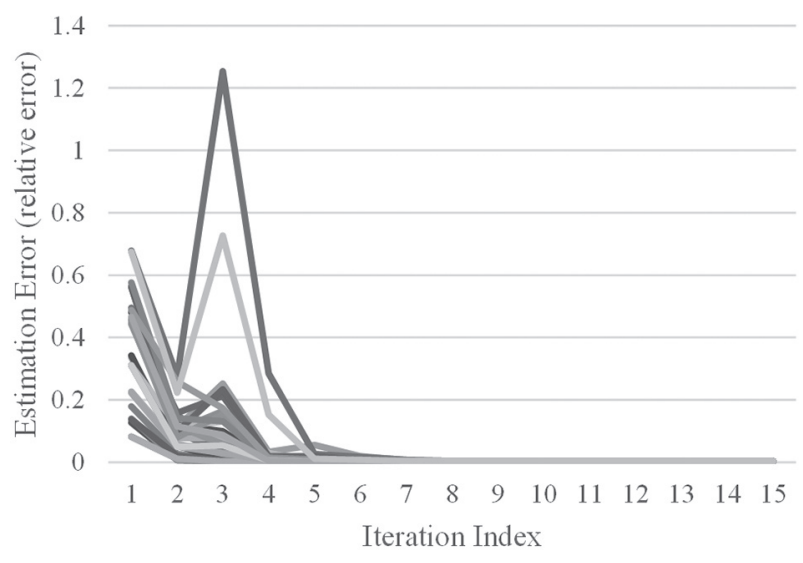

Fig. 4. Convergence of the estimation using the bound-constrained optimization method over 30 initial guesses

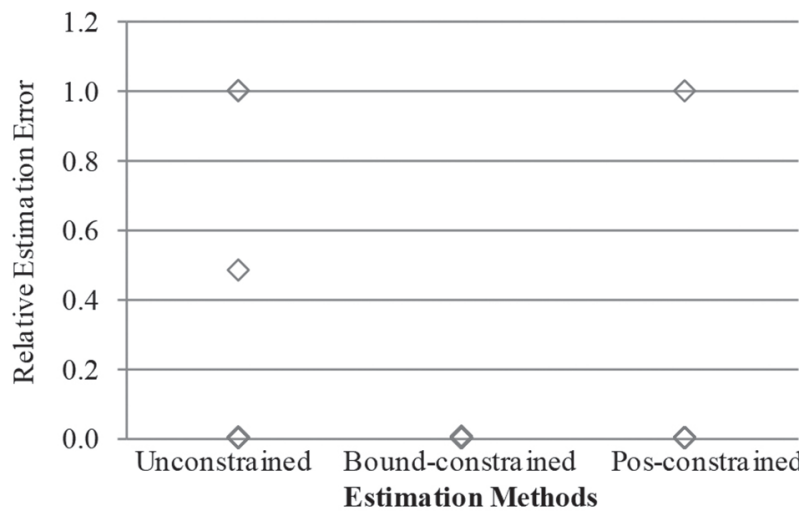

Fig. 6. Estimation errors using the unconstrained, the bound-constrained, and the positivityconstrained optimization method over 30 initial guesses

This can be used to explain the better convergence performance of the positivity-constrained optimization method, as shown in figures 4 and 5, because the lower condition number will result in less susceptibility to noise and error (the error in this case is the linearization error due to the different selection of initial guesses). Considering the change of region sensitivity, as shown in Table 4 and Figure 7, significant decreases in sensitivity are noticeable in the scalp and the skull region. In the unconstrained case, the sensitivity of the skull region is the largest. It is 53 times larger than that of the scalp region and it is 419-1,806 times larger than that of the inner regions. These numbers are completely different after constraining. In the bound-constrained case, the sensitivity of the skull is larger than that of the scalp and those of the inner tissues by only 2.4 times and only 1354 times, respectively. In the positivity-constrained case, the sensitivity of the skull is larger than that of the scalp and the inner regions by only 2 times and only 8-22 times, respectively. There is no significant difference between the two constrained optimization methods.

The very large sensitivity of the skull region in the unconstrained case resulted in a very large sensitivity 
to noise and error of skull conductivity estimation. The accuracy of skull conductivity estimation was then not accurate. The very small sensitivity of the inner regions compared to the sensitivity of the scalp and the skull regions caused a very small change in conductivity value from the initial values. In other words, even though the difference between the exact conductivity value and the initial guess value is large, it will result in a very small change from the initial value and then slightly impact the estimation. In the case of the CSF, GM, and WM, even though the improvement of sensitivity was carried out by constraining, the accuracy of CSF, GM, and WM conductivity estimation was not significantly improved in general. This may indicate the insufficiently large sensitivity of these inner regions. Furthermore, surprisingly, poor accuracy of skull conductivity estimation and many over-range estimates of the CSF conductivity were found in the case of using the positivity-constrained optimization method. This is due to both the reduction of the error in the case of skull conductivity estimation and the enlargement of the error in the case of CSF conductivity estimation after transforming $\gamma$ back to $\sigma$. Considering $\sigma-\gamma$ mapping of the positivity-constrained case in Fig. 8, a very large error in $\gamma$ of the skull conductivity becomes a very small error in $\sigma$ when it is reversely transformed back. Therefore, the conductivity value of the skull conductivity will only slightly change from the initial value even though the error is large. On the other hand, a very small error in $\gamma$ of the CSF conductivity becomes a very large error in $\sigma$. In contrast, a large change in the conductivity value will occur in every iteration of CSF conductivity estimation. Therefore, the robustness for CSF conductivity estimation is poor. A degree of complexity and nonlinearity of the system is then substantially increased by positivity-constraining. Compared to the case of using the bound-constrained optimization method, these phenomena will occur with a lesser degree in the range of conductivity values used, and this causes the bound-constrained optimization method to be more robust and more accurate than the others.

Table 3. Condition number of the Jacobian matrices ${ }^{\mathrm{a}}$

$\begin{array}{ccc}\text { Unconstrained method } & \begin{array}{c}\text { Bound- } \\ \text { constrained method }\end{array} & \text { Positivity-constrained method } \\ 61 \times 106 \pm 14 \times 106 & 1.95 \times 106 \pm 0.41 \times 106 & 0.80 \times 106 \pm 0.14 \times 106\end{array}$

Table 4. Norm of sensitivities in each tissue region ${ }^{a}$

\begin{tabular}{lccc} 
Tissues & $\begin{array}{c}\text { Unconstrained method } \\
\text { (V.m/S) }\end{array}$ & $\begin{array}{c}\text { Bound-constrained method } \\
\text { (V.m/S) }\end{array}$ & $\begin{array}{c}\text { Positivity-constrained } \\
\text { method (V.m/S) }\end{array}$ \\
\hline Scalp & $5.492 \pm 1.111$ & $1.668 \pm 0.172$ & $2.062 \pm 0.171$ \\
Skull & $288.981 \pm 59.72$ & $4.080 \pm 0.475$ & $4.109 \pm 0.476$ \\
CSF & $0.160 \pm 0.026$ & $0.076 \pm 0.019$ & $0.185 \pm 0.007$ \\
GM & $0.535 \pm 0.102$ & $0.161 \pm 0.015$ & $0.199 \pm 0.014$ \\
\hline WM & $0.690 \pm 0.056$ & $0.325 \pm 0.026$ & $0.524 \pm 0.043$
\end{tabular}

a The value represented as the mean \pm standard

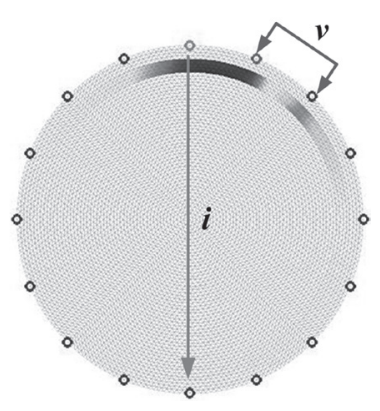

(a) Unconstrained optimization method

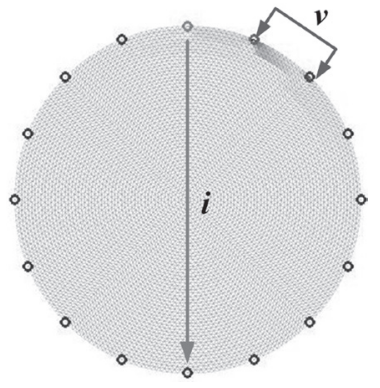

(b) Bound-constrained optimization method

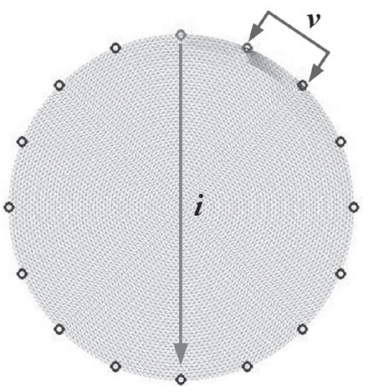

(c) Positivity-constrained optimization method

Fig. 7. An example of the sensitivity of the measurement $v$ to the applied current $i$ of the unconstrained, the bound-constrained, and the positivity-constrained optimization methods 


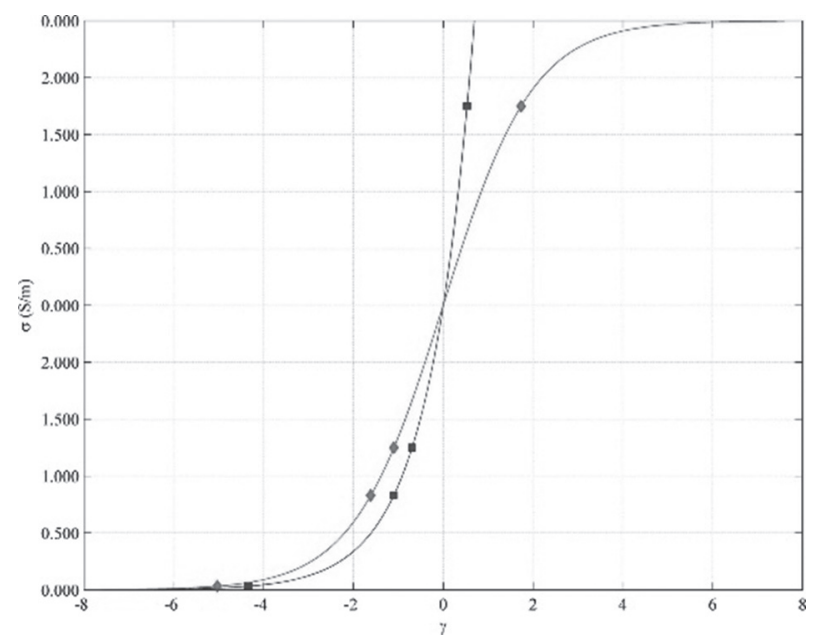

Fig. 8: $\sigma-\gamma$ Mapping of the bound-constraint implementation (the red line) and the positivityconstraint implementation (the blue line)

\section{CONCLUSION}

In this study, the estimation performance of the unconstrained optimization method and two constrained optimization methods, i.e. the bound-constrained and the positivity-constrained optimization method for estimating the scalp, the skull, the CSF, the GM, and the WM conductivity were investigated. The use of constraints significantly improved the sensitivity of the scalp and the skull region, and it resulted in the improvement of the accuracies and the robustness of estimation, even though it increased a degree of nonlinearity of estimation. Estimation with the positivityconstraint optimization method, however, resulted in an unexpected outcome for the estimation of the CSF and the skull conductivity due to a significant increase in the nonlinearity degree. Therefore, only the boundconstrained optimization is advisable here for head tissue conductivity estimation. It is also good to note that the sensitivity of the CSF, the GM, and the WM region was probably too small to obtain accurate conductivities of these tissues.

\section{REFERENCES}

[1] T. Tidswell, A. Gibson, R. H. Bayford, and D. S. Holder, "Three-Dimensional Electrical Impedance Tomography of Human Brain Activity", Neurolmage, Vol. 13, No. 2, 2011, pp. 283-294.

[2] J. D. Johansson, P. Blomstedt, N. Haj-Hosseini, T. Bergenheim, O. Eriksson, and K. Wårdell, "Combined diffuse light reflectance and electric impedance measurements for navigation aid in deep brain surgery", Stereotact Funct Neurosurg, Vol. 87, No.2, 2009, pp. 105-113.

[3] J. Vorwerk, J.-H. Cho, S. Rampp, H. Hamer, T. R. Knösche, and C. H.Wolters, "A guideline for head volume conductor modeling in EEG and MEG", Neurolmage, Vol. 100, October, 2014, pp. 590-607.

[4] J. Latikka, T. Kuurne, and H. Eskola, "Conductivity of living intracranial tissues", Phys. Med. Biol., Vol. 46, No. 6, 2001, pp. 1611-1616.

[5] L. Koessler, S. Colnat-Coulbois, T. Cecchin, J. Hofmanis, J. P.Dmochowski, A. M.Norcia, and L. G.Maillard, "In-Vivo Measurements of Human Brain Tissue Conductivity Using Focal Electrical Current Injection Through Intracerebral Multicontact Electrodes", Human Brain Mapping Vol. 38, No. 2, 2017, pp. 974-986.

[6] S. I. Gonçalves, J. C. de Munck, J. P. A. Verbunt, F. Bijma, R. M. Heethaar, and F. L. d. Silva, "In Vivo Measurement of the Brain and Skull Resistivities Using an EIT-Based Method and Realistic Models for the Head", IEEE Transactions on Biomedical Engineering, Vol. 50, No. 6, 2003, 754-767

[7] U. Baysal and J. Haueisen, "Use of a priori information in estimating tissue resistivities-application to human data in vivo", Physiol. Meas., Vol. 25, No. 3, 2004, pp. 737-748.

[8] T. F. Oostendorp and J. Delbeke, "The conductivity of the human skull in vivo and in vitro", presented at the Engineering in Medicine and Biology Conference, 21st Annual Conf. and the 1999 Annual Fall Meeting of the Biomedical Engineering Soc. BMES/EMBS Conference, Atlanta, 1999.

[9] M. Fernández-Corazza, S. Turovets, P. Govyadinov, C. H. Muravchik, and D. Tucker, "Effects of Head Model Inaccuracies on Regional Scalp and Skull Conductivity Estimation Using Real EIT Measurements", II Latin American Conference on Bioimpedance, IFMBE Proceedings, Vol. 54, 2016., pp 5-8

[10] T. Ouypornkochagorn, N. Polydorides, and H. McCann, "In Vivo Estimation of the Scalp and Skull Conductivity", presented at the 15th International Conference on Biomedical Applications of Electrical Impedance Tomography, Gananoque, Ontario, Canada, 2014., p. 10

[11] M. Dannhauer, B. Lanfer, C. H. Wolters, and T. R. Knosche, "Modeling of the human skull in EEG source analysis", Human Brain Mapping, Vol. 32, No. 9, 2011, pp. 1383-1399. 
[12] N. Polydorides, T. Ouypornkochagorn, and H. McCann, "Inequality Constrained EIT Modelling and Inversion", presented at the $15_{\text {th }}$ International Conference on Biomedical Applications of Electrical Impedance Tomography, Gananoque, Ontario, Canada, 2014., p. 89

[13] T. Yamamoto and Y. Yamamoto, “Electrical properties of the epidermal stratum corneum", Med. Biol. Eng., Vol. 14, No. 2, 1976, pp. 494-500.

[14] H. C. Burger and J. B. v. Milaan, "Measurements of the specific resistance of the human body to direct current", Acta Medica Scandinavica, Vol. CXIV, 1943.

[15] M. Akhtari, H. C. Bryant, A. N. Mamelak, E. R. Flynn, L. Heller, J. J. Shih, M. Mandelkern, A. Matlachov, D. M. Ranken, E. D. Best, M. A. DiMauro, R. R. Lee, and W. W. Sutherling, "Conductivities of Three-Layer Live Human Skull", Brain Topography, Vol. 14, No. 3, 2002, pp. 151-167.

[16] S. K. Law, "Thickness and resistivity variations over the upper surface of the human skull", Brain Topography, Vol. 6, No. 2, 1993, pp. 99-109.
[17] R. Hoekema, G. H. Wieneke, F. S. S. Leijten, C. W. M. v. Veelen, P. C. v. Rijen, G. J. M. Huiskamp, J. Ansems, and A. C. v. Huffelen, "Measurement of the Conductivity of Skull, Temporarily Removed During Epilepsy Surgery", Brain Topography, Vol. 16, No. 1, 2003, pp. 29-38.

[18] J. D. Kosterich, K. R. Foster, and S. R. Pollack, "Dielectric permittivity and electrical conductivity of fluid saturated bone", IEEE Trans. Biomed. Eng, Vol. 30, No. 2, 1983, pp. 81-86.

[19] S. B. Baumann, D. R. Wozny, S. K. Kelly, and F. M. Meno, "The Electrical Conductivity of Human Cerebrospinal Fluid at Body Temperature", IEEE Transactions on Biomedical Engineering, Vol. 44, No. 3, 1997, pp. 220-223.

[20] N. Polydorides, "Image Reconstruction Algorithms for Soft-field Tomography", PhD thesis, The University of Manchester Institute of Science and Technology, 2002. 